\title{
COVID-19, embarazo, vacunas y lactancia materna
}

\section{COVID-19, pregnancy, vaccines and breastfeeding}

\author{
Dr. Eduardo López Mora ${ }^{1}$ \\ Dr. Jorge Espinoza Rojas ${ }^{2}$ \\ Dra. Jeannette Dabanch Peña ${ }^{3,4}$ \\ Dr. Rodrigo Cruz Choappa ${ }^{*}$ \\ ${ }^{1}$ Residente Infectología Universidad de Valparaíso. Hospital San Camilo. \\ ${ }^{2}$ Residente Infectología Universidad de Valparaíso. \\ ${ }^{3}$ Hospital Clínico de la Universidad de Chile \\ ${ }^{4}$ Centro de Diagnóstico e Investigación de Enfermedades Infecciosas (CDIEI) \\ Universidad de Valparaíso \\ *Autor para correspondencia: rodrigo.cruz@uv.cl
}

RECIBIDO: 24 de mayo 2021

APROBADO: 2 de junio 2021

DOI: 10.22370/bolmicol.2021.36.1.2854

\section{RESUMEN}

Las mujeres embarazadas o en período de lactancia han sido excluidas de los ensayos clínicos sobre vacunas contra SARS-CoV-2, evitando así la obtención de datos sólidos que permitan determinar la seguridad e inmunogenicidad de las vacunas en esta población, a la vez que se han asociado peores resultados maternos - fetales.

La evidencia acerca de la seguridad e inmunogenicidad en esta población es limitada, en base a estudios observacionales, con pocos casos y en mujeres vacunadas con plataformas ARNm, en las cuales no se ha descrito por ahora una mayor asociación a eventos adversos relacionados a vacunas, como tampoco, variaciones significativas en la respuesta inmunológica en comparación a la población no embarazada. También existen datos que documentan la adquisición de anticuerpos transplacentarios, considerándose de bajo riesgo la posibilidad de transmisión vertical. Se hacen necesarios ensayos clínicos que permitan precisar recomendaciones basadas en evidencia para esta población, en un contexto de utilización de emergencia de vacunas contra SARS-CoV-2.

\section{ABSTRACT}

Pregnant or breastfeeding women have been excluded from clinical trials on vaccines against SARSCoV-2, thus avoiding obtaining solid data to determine the safety and immunogenicity of vaccines in this population, as well as being associated worse maternal-fetal outcomes. 
COVID-19, embarazo, vacunas y lactancia materna - López E.

The evidence about safety and immunogenicity in this population is limited, based on observational studies, with few cases and in women vaccinated with mRNA platforms, in which a greater association to adverse events related to vaccines has not been described or significant variations in the immune response compared to the non-pregnant population. There are also data that document the acquisition of transplacental antibodies, considering the possibility of vertical transmission as low risk. Clinical trials are necessary to evidence-based recommendations for this population, in a context of emergency use of vaccines against SARSCoV-2.

\section{INTRODUCCIÓN}

Al 1 de junio del 2021 el virus SARS-CoV-2 ha causado más de 170 millones de infecciones y más de 3,5 millones de muertes en todo el mundo (1). A la fecha, la OMS ha aprobado el uso de emergencia de 7 vacunas, que provienen de 3 plataformas distintas, sin embargo, hay más de 100 vacunas en fase de desarrollo clínico (2). Se estima en más de 835 millones de personas las vacunadas con al menos 1 dosis en todo el mundo (3).

Las mujeres embarazadas o en período de lactancia han sido excluidas de los ensayos clínicos sobre vacunas contra SARS-CoV-2, evitando así la obtención de datos sólidos que permitan determinar la seguridad e inmunogenicidad de las vacunas en esta población, por otra parte, hay datos que asocian peores resultados maternos fetales, considerándose como una población de mayor riesgo. Las entidades reguladoras, asesoras y sociedades científicas, en su mayoría proveen recomendaciones que llaman a considerar la vacunación, tomando en cuenta el riesgo de exposición y tras un proceso de toma de decisión informada por parte de la paciente.

\section{Cambios fisiológicos del embarazo y COVID- 19}

El embarazo se caracteriza por una serie de cambios fisiológicos, que prácticamente inciden en todos los sistemas. En relación a la infección por SARS-CoV-2 y la evolución a COVID-19 severo, hay dos sistemas fundamentales en la respuesta a COVID-19; la respuesta inmunológica y la reserva funcional respiratoria (4).

En lo que respecta a la respuesta inmunológica, se incluyen fenómenos bien documentados como el desarrollo de anticuerpos neutralizantes, respuesta a través de Linfocitos T e interferón gamma, además de la transferencia transplacentaria de anticuerpos y el potencial de transmisión vertical. Durante el embarazo existe además un aumento del consumo de oxígeno y una disminución del volumen residual funcional generando una disminución de la reserva materna de $\mathrm{O} 2$, además de una menor capacidad de compensar la acidosis, lo cual hace a la embarazada más susceptible a la hipoxia y el shock (5).

\section{Resultados maternos - fetales en COVID-19}

Los datos actuales siguen siendo escasos y provienen de estudios observaciones. La evidencia actual no muestra un aumento en la susceptibilidad a la infección por SARS-CoV-2 en esta población, sin embargo, sí se consideran un grupo de alto riesgo, dada la asociación a mayor incidencia de enfermedad grave, ingreso a UCI, ventilación mecánica y muerte. En un reporte de datos que provienen del sistema de vigilancia nacional en los EEUU, que incluye a cerca de 1,3 millones de mujeres en edad fértil con infección con SARS-CoV-2, de las cuales en poco más de 23 mil se contaba con el dato de embarazo, COVID-19 se asoció a peores resultados maternos, aunque los números 
COVID-19, embarazo, vacunas y lactancia materna - López E.

absolutos de resultados graves siguen siendo bajos; Ingreso a UCI 3 veces mayor RR (10,5 frente a 3,9 x cada 1000 casos), requerimiento de ventilación mecánica invasiva $(R R=2.9)$, necesidad de ECMO $(\mathrm{RR}=2,4)$ y muerte $(\mathrm{RR}=$ 1,7). Además, hubo ciertas condiciones que se asociaron a mayor riesgo de resultados adversos, tales como; edad $\geq 35$ años, obesidad, comorbilidades médicas preexistentes (en particular hipertensión y diabetes) (6).

En relación al resultado perinatal, hay datos que han reportado mayor riesgo de parto prematuro pero no de aborto espontáneo, ni mortinatos, ni de anomalías congénitas. Datos publicados por los Centros de Prevención y Control de Enfermedades (CDC) de EEUU, los cuales provienen del Sistema Nacional de Vigilancia de Enfermedades Notificables. Sobre 5.252 embarazos, de los cuales 3.912 fueron nacidos vivos con edad gestacional conocida, el $12,9 \%$ fueron prematuros, mayor al 10,2\% entre la población general de EEUU en 2019. Entre 610 recién nacidos $(21,3 \%)$ con resultados de PCR para SARS-CoV-2, la infección perinatal fue poco frecuente $(2,6 \%)$. (7). En un estudio publicado recientemente, que incluyó el $92 \%$ de los embarazos en Suecia durante los primeros 10 meses de la pandemia, los hijos de madres positivas al SARS-CoV-2 tuvieron un pequeño aumento en la tasa de parto prematuro $(8,8 \%$ versus $5,5 \%)$ y de cualquier trastorno respiratorio en comparación con los lactantes de madres no infectadas ( 2.8 versus 2,0 por ciento) (8).

\section{Riesgo de transmisión vertical}

Sigue aún no bien dilucidado su impacto. Sólo se han publicado pocos casos bien documentados de probable transmisión vertical. Se debe considerar que la viremia en pacientes con COVID-19 parecen ser transitorias y bajas, pero significativamente más alta en la enfermedad grave. En una revisión sistemática que evaluó transmisión vertical a través de la detección de ARN para SARS-CoV-2 en fetos y neonatos, hasta 48 hrs de nacidos, además de evaluar su presencia en cordón umbilical, placenta y líquido amniótico, se incluyeron estudios observacionales que acumulan un total 979 muestras, de las cuales en solo un 3,2 \% (27 casos) se detectó ARN para SARS-CoV-2. En muestras de sangre del cordón umbilical fue positiva en el $2.9 \%$ de las muestras (1/34), el $7.7 \%$ de las muestras de placenta $(2 / 26)$, y no hubo evidencia de ARN viral en líquido amniótico (0/51) (9).

En otro estudio de cohorte multicéntrico que incluyó a 63 mujeres con PCR positivo para SARS-CoV-2, se estudió ARN en cordón umbilical, placenta y en sangre del recién nacido. No hubo casos de transmisión vertical, tampoco hubo ARN detectable en sangre de cordón, ni placenta (10).

La vía de transmisión postnatal puede ocurrir a través de secreciones respiratorias de la madre infectada $\mathrm{u}$ otro cuidador. Se ha detectado ARN para SARS-CoV-2 en la leche materna con resultados disimiles entre estudios. No hay evidencia hasta el momento de virus viable en leche materna. La recomendación de American Academy of Pediatrics (AAP) es mantener la lactancia materna (11).

\section{Inmunogenicidad y seguridad de las vacunas contra SARS-CoV-2 en el embarazo y periodo de lactancia}

Actualmente, existe gran controversia en el mundo acerca de la seguridad de las vacunas contra SARS-CoV-2 en el embarazo, considerando la ausencia de estudios publicados fase II / III en esta población y por otro lado la actual autorización de emergencia para el uso de 
COVID-19, embarazo, vacunas y lactancia materna - López E.

vacunas por las entidades reguladoras. Pese a lo expuesto hay algunos datos promisorios que provienen de estudios observacionales. En una cohorte prospectiva (no revisada por pares), que incluyó 131 mujeres que recibieron vacunas ARNm; 84 embarazadas, 31 en lactancia y 16 no embarazadas, se evaluaron títulos de anticuerpos contra la proteína Spike y contra el dominio de unión a receptor (RBD) $\operatorname{IgG}, \operatorname{Ig} \mathrm{A}$ e $\operatorname{IgM}$, al inicio y 2 semanas después de la segunda dosis de vacuna en el suero de todas las mujeres. Además, se estudió la presencia de anticuerpos en muestras provenientes de cordón umbilical $(\mathrm{N}=10)$ y leche materna $(\mathrm{N}=31)$. Los títulos se compararon con mujeres embarazadas de 4 a 12 semanas después de la infección natural $(\mathrm{N}=$ 37). No hubo diferencia significativa en los títulos posterior a la vacunación entre embarazadas, mujeres en periodo de lactancia y no embarazadas. En los resultados es destacable que los títulos de anticuerpos fueron significativamente más altos en las pacientes vacunadas que en aquellas con antecedente de infección por SARS-CoV-2 durante el embarazo. En todos los casos se detectaron anticuerpos en sangre del cordón umbilical y leche materna. No hubo diferencias significativas en eventos adversos severos, por lo tanto, se desprende de este estudio que las vacunas de ARNm de COVID-19 se asociaron a presencia de anticuerpos en mujeres embarazadas y en periodo de lactancia, con inmunogenicidad similar en mujeres no embarazadas, con eventos adversos similares, debiendo considerar el número reducido de participantes. También las respuestas inmunitarias inducidas por la vacuna fueron significativamente mayores que la respuesta a la infección natural, fenómeno a esta altura bien documentado (12).

En otra cohorte prospectiva desarrollada en Israel entre diciembre del 2020 y marzo del 2021 en la que se buscó evaluar la inmunogenicidad de vacunas de ARNm en mujeres embarazadas y en lactancia incluso frente a variantes de preocupación de SARS-CoV- 2. El estudio incluyó 103 mujeres que recibieron alguna vacuna ARNm: 30 mujeres embarazadas, 16 en periodo de lactancia y 57 que no estaban embarazadas, por otra parte, también se incluyó de un total de 28 mujeres que se confirmó infección por SARS-CoV-2 (22 mujeres embarazadas y 6 no embarazadas), durante un periodo de tiempo similar. En todos los grupos mencionados se evaluaron títulos de anticuerpos contra el dominio de unión a receptor (RBD), respuesta de LT específicas contra proteína Spike (tinción de citoquinas intracelulares e inmunospot para detección IFN-gama) contra cepa original circulante SARS-CoV-2 USAWA1 / 2020 y variantes B.1.1.7 y B.1.351. En los resultados destacó que en los títulos de anticuerpos anti RBD neutralizantes y no neutralizantes funcionales no hubo diferencias significativas entre las poblaciones. Las respuestas de células $\mathrm{T}$ CD4+ y CD8+, estuvieron presentes en mujeres embarazadas, en periodo de lactancia y no embarazadas después de la vacunación. Se detectaron anticuerpos anti RBD en la sangre del cordón umbilical y la leche materna. In vitro hubo reducción significativa de la neutralización por anticuerpos contra las variantes B.1.1.7 y B.1.351, aunque las respuestas a través de LT se conservaron contra las variantes de preocupación (13).

En otros estudios observacionales, con importantes limitaciones, no se han informado diferencias significativas en eventos adversos frente a embarazadas no vacunadas. Datos que se desprenden de un total de 94.000 participantes en V-safe, sistema de seguimiento activo posterior a la vacunación de los CDC de EEUU, donde se reportan datos de 827 embarazos completos, vacunadas principalmente en el tercer trimestre, sin evidencia de eventos adversos perinatales distintos a población no 
COVID-19, embarazo, vacunas y lactancia materna - López E.

vacunada, como aborto espontáneo, anomalías congénitas, parto prematuro, muerte fetal o muerte neonatal. El sistema de informes de eventos adversos de vacunas de los CDC de EEUU, también reportó datos sobre 154 embarazos, sin diferencias significativas en comparación con los datos nacionales de nacimientos (14).

\section{DISCUSIÓN}

En la actualidad no existen estudios Fase II/III en población embarazada o en periodo de lactancia. Durante este periodo de pandemia se han desarrollado una serie de estudios en su mayoría observacionales que han intentado responder a estas interrogantes, de las cuales, se cuentan con datos, provenientes de mujeres inoculadas con vacunas de plataforma ARNm, las que predominan en EEUU y Europa. En Chile, a la fecha están autorizadas 4 vacunas; CoronaVac (Sinovac Life Sciences), BNT162b2 (PfizerBioNTech), AZD1222/ChAdOx1-S (Oxford-AstraZeneca) y en los últimos días Ad5-nCoV/ Convidencia ${ }^{\circledR}$ (Cansino Biologics Inc), de las cuales solo existen reportes en estudios observacionales de BNT162b2.

Las entidades reguladoras, asesoras y sociedades científicas, en todo el mundo, han ido actualizando sus recomendaciones a la luz de la vertiginosa producción de información acerca de vacunas contra SARS-CoV-2, sin embargo, actualmente las recomendaciones en embarazo y periodo de lactancia siguen siendo controversiales, en el contexto actual de aprobación de uso de emergencia y la ausencia de datos sólidos que permitan recomendar una vacunación universal. La evaluación del riesgobeneficio, considerando la probabilidad de exposición y de desenlaces negativos deben ser la base de una decisión conjunta entre paciente y equipo tratante. El distanciamiento social, los elementos de protección personal, en conjunto a una estrategia adecuada de comunicación de riesgo y optimizar la vacunación de la población, parecieran ser claves para frenar la propagación.

Conflicto de interés: los autores no tienen conflictos de interés.

Financiamiento: Universidad de Valparaíso.

\section{REFERENCIAS}

1. WHO Coronavirus (COVID-19) Dashboard. June 2021. https://covid19.who.int/

2. WHO. Novel Coronavirus_Landscape_COVID-19.

June 2021. https://www.who.int/publications/m/ite m.

3. Our World in Data COVID-19 dataset. June 2021. https://ourworldindata.org/covidvaccinations.

4. Karimi L, Makvandi S, Vahedian-Azimi A, Sathyapalan T, Sahebkar A. Effect of COVID-19 on Mortality of Pregnant and Postpartum Women: A Systematic Review and Meta-Analysis. J Pregnancy. 2021. doi: $10.1155 / 2021 / 8870129$

5. Hegewald M, Crapo R. Respiratory physiology in pregnancy. Clin Chest Med. $2011 \quad$ Mar;32(1):1-13. DOI: $\underline{10.1016 / \text { j.ccm.2010.11.001. }}$.

6. Zambrano L, Ellington S, Strid P, Galang RR, Oduyebo T, Tong V, Woodworth K, 
COVID-19, embarazo, vacunas y lactancia materna - López E.

et.al. CDC COVID-19 Response Pregnancy and Infant Linked Outcomes Team. Update: Pregnancy Status 2020. MMWR Morb Mortal Wkly Rep. 2020. https://www.cdc.gov/mmwr/volumes/69 /wr/mm6944e3.htm.

7. Woodworth K, Olsen E, Neelam V, Lewis E, Galang R, Oduyebo T, et al. CDC. COVID-19 Pregnancy and Infant Linked Outcomes Team (PILOT). Birth and Infant Outcomes Following Laboratory-Confirmed SARS-CoV-2 Infection in Pregnancy - SET-NET. MMWR Morb Mortal Wkly Rep. 2020. https://www.cdc.gov/mmwr/volumes/69 /wr/mm6944e2.htm.

8. Norman M, Navér L, Söderling J, Ahlberg M, Hervius Askling $\mathrm{H}$, Aronsson B, et al. Association of Maternal SARS-CoV-2 Infection in Pregnancy With Neonatal Outcomes. JAMA. 2021. doi: 10.1001 / jama.2021.5775.

9. Kotlyar A, Grechukhina O, Chen A, Popkhadze S, Grimshaw A, Tal O, et al. Vertical transmission of coronavirus disease 2019: a systematic review and meta-analysis. Am J Obstet Gynecol. 2021. doi: $10.1016 /$ j.ajog.2020.07.049.

10. Edlow A, Li J, Collier A, Atyeo C, James $\mathrm{K}$, Boatin A, et al. Assessment of Maternal and Neonatal SARS-CoV-2 Viral Load. JAMA New Open. 2020. doi: $\quad 10.1001$ jamanetworkopen.2020.30455.

11. American Academy of Pediatrics. FAQs: Management of Infants Born to COVID19 Mothers https://services.aap.org/en/pages/2019- novel-coronavirus-covid-19infections/clinical-guidance/faqsmanagement-of-infants-born-to-covid19-mothers.

12. Gray K, Bordt E, Atyeo C, Deriso E, Akinwunmi B, Young N, et al. COVID19 vaccine response in pregnant and lactating women: a cohort study. Am J Obstet Gynecol. 2021. doi.org/10.1016/j.ajog.2021.03.023.

13. Collier A, McMahan K, Yu J, Tostanoski L, Aguayo R, Ansel J, et al. Immunogenicity of COVID-19 mRNA Vaccines in Pregnant and Lactating Women. JAMA. 2021. doi: 10.1001 / jama.2021.7563

14. Shimabukuro T, Kim S, Myers T, Moro $\mathrm{P}$, Oduyebo T, Panagiotakopoulos L, et al. CDC v-safe COVID-19 Pregnancy Registry Team. Preliminary Findings of mRNA Covid-19 Vaccine Safety in Pregnant Persons. N Engl J Med. 2021. DOI: 10.1056 / NEJMoa2104983. 